\title{
The Frobenius problem for the shuffle operation
}

\author{
Jeremy Nicholson* and Narad Rampersad ${ }^{\dagger}$ \\ Department of Mathematics and Statistics \\ University of Winnipeg \\ 515 Portage Avenue \\ Winnipeg, Manitoba R3B 2E9 (Canada) \\ jnich998@hotmail.com,n.rampersad@uwinnipeg.ca
}

September 20, 2018

\begin{abstract}
Given a set $S$ of words, let $S^{\dagger}$ denote the iterated shuffle of $S$. We characterize the finite sets $S$ for which $S^{\dagger}$ is co-finite, and we give some bounds on the length of a longest word not in $S^{\dagger}$.
\end{abstract}

\section{Introduction}

The classical Frobenius problem is the following: Given positive integers $m_{1}, m_{2}, \ldots, m_{k}$ such that $\operatorname{gcd}\left(m_{1}, m_{2}, \ldots, m_{k}\right)=1$, what is the largest integer that cannot be written as a non-negative integer linear combination of $m_{1}, m_{2}, \ldots, m_{k}$ ? Schur showed that this number always exists and Sylvester showed that when $k=2$ this number is equal to $m_{1} m_{2}-m_{1}-m_{2}$. The case $k \geq 3$ is rather more difficult. An entire book has been devoted to this problem [6].

Shallit proposed the following "non-commutative" version of the Frobenius problem: Given a set of words $S=\left\{w_{1}, w_{2}, \ldots, w_{k}\right\}$ over an alphabet $\Sigma$ such that $S^{*}$ is co-finite (i.e., contains all but finitely many words over $\Sigma$ ), what is the length of a longest word not in $S^{*}$ ? In other words, what is the length of a longest word that cannot be written as a concatenation of a sequence of words chosen from $S$ ? Xu studied this problem, which he called the Frobenius problem in the free monoid, in his Ph.D. thesis [8]. Note that this problem reduces to the classical Frobenius problem when $\Sigma$ is a unary alphabet. For larger alphabets, Xu considered the special case where $S$ contains words of only two lengths $m$ and $n$ (say, $m<n$ ). He showed that the answer to the problem in this setting could be exponential in $n-m$.

In this paper we examine the same problem with respect to the shuffle operation on words. Informally, the shuffle of two words $u$ and $v$ is the set of all words that can be obtained by "interleaving" the letters of $u$ with the letters of $v$ in all possible ways. This shuffle

${ }^{*}$ The author was supported by an NSERC USRA.

${ }^{\dagger}$ The author was supported by NSERC Discovery Grant No. 418646-2012. 
operation on words was introduced, in an algebraic setting, by Eilenberg and MacLane [2]. Some notable early papers that study the shuffle operation from a formal languages perspective are Jantzen [3, 4] and Warmuth and Haussler [7].

Given a set $S$ of words, let $S^{\dagger}$ denote the iterated shuffle of $S$ (see the formal definition in the next section). In this paper we characterize the finite sets $S$ for which $S^{\dagger}$ is co-finite, and we show that the length of a longest word not in $S^{\dagger}$ is at most quadratic in the length of the longest word in $S$. The Frobenius problem in this setting therefore turns out to be somewhat closer to the classical integer version of the problem, rather than the "free monoid" version of the problem studied by $\mathrm{Xu}$.

\section{Preliminaries}

Let us recall again the classical Frobenius problem: Given positive integers $m_{1}, m_{2}, \ldots, m_{k}$ such that $\operatorname{gcd}\left(m_{1}, m_{2}, \ldots, m_{k}\right)=1$, what is the largest integer that cannot be written as a non-negative integer linear combination of $m_{1}, m_{2}, \ldots, m_{k}$ ? Let $g\left(m_{1}, m_{2}, \ldots, m_{k}\right)$ denote this quantity, which is known as the Frobenius number for the given instance of the problem. For $k=2$ we have the exact formula of Sylvester: $g\left(m_{1}, m_{2}\right)=m_{1} m_{2}-m_{1}-m_{2}$. For $k \geq 2$ there are a number of upper bounds; for our purposes, the following one due to Schur (see [1]) will suffice: if $m_{1} \leq m_{2} \leq \cdots \leq m_{k}$, then

$$
g\left(m_{1}, m_{2}, \ldots, m_{k}\right) \leq m_{1} m_{k}-m_{1}-m_{k} .
$$

Our goal in this paper is to generalize the Frobenius problem to the setting of words over an alphabet. Let $\Sigma$ denote an alphabet and let $\Sigma^{*}$ denote the set of all words over $\Sigma$. For any $a \in \Sigma$ and $w \in \Sigma^{*}$, the number of occurrences of $a$ in $w$ is denoted by $|w|_{a}$.

Let $|\Sigma|=q$ and let $S$ be a finite subset of $\Sigma^{*}$ such that $S^{*}$ is co-finite. Xu showed that if $q>1$ and $S$ contains words of lengths $m$ and $n$ only $(m<n)$, then the longest word not in $S^{*}$ has length at most $m q^{n-m}+n-m$ and this bound is tight.

The Kleene star operator used above can be viewed as iterated concatenation. In this paper we will study the Frobenius problem for iterated shuffle. The shuffle operator can be defined as follows

$$
u \amalg v=\left\{u_{1} v_{1} u_{2} v_{2} \cdots u_{k} v_{k}: u_{i}, v_{i} \in \Sigma^{*}, u=u_{1} u_{2} \cdots u_{k}, \text { and } v=v_{1} v_{2} \cdots v_{k}\right\}
$$

The iterated shuffle1 of a word is defined by

$$
u^{\dagger}=\bigcup_{i=0}^{\infty} \underbrace{(u \sqcup u \sqcup \cdots \sqcup u)}_{i \text { times }} .
$$

We extend both of these operations from words to sets of words in the usual way. First, for sets of words $A$ and $B$ we define

$$
A \sqcup B=\{u \sqcup v: u \in A \text { and } v \in B\},
$$

\footnotetext{
${ }^{1}$ There is no standard notation to denote the iterated shuffle; we are following Jantzen's use of the "dagger".
} 
and

$$
A^{\dagger}=\bigcup_{i=0}^{\infty} \underbrace{(A \sqcup A \sqcup \cdots \sqcup A)}_{i \text { times }} .
$$

For example,

$$
\{01,011\} \sqcup\{0\}=\{001,010,0011,0101,0110\}
$$

and

$$
\{01,2\}^{\dagger}=\left\{w \in\{0,1,2\}^{*}:|w|_{0}=|w|_{1} \text { and every prefix } u \text { of } w \text { satisfies }|u|_{0} \geq|u|_{1}\right\} .
$$

Over the alphabet $\{0,1\}$ the set $\{00,000,11,111,01,10\}^{\dagger}$ is co-finite; the only strings not in this set are $0,1,001,010,100,011,101,110$.

The Frobenius problem for the shuffle operation is therefore the following: If $S$ is a finite set of words over $\Sigma$ such that $S^{\dagger}$ is co-finite, what is the length of a longest word not in $S^{\dagger}$ ? Note that when $|\Sigma|=1$, say $\Sigma=\{x\}$, this problem is equivalent to the integer Frobenius problem. In this case, either $x \in S$, in which case $S^{\dagger}=\Sigma^{*}$, or $S$ has the form $S=\left\{x^{m_{1}}, x^{m_{2}}, \ldots, x^{m_{k}}\right\}$, where $m_{i} \geq 2$ for $i=1,2, \ldots, k$. The length of any word in $S^{\dagger}$ is a multiple of $\operatorname{gcd}\left(m_{1}, m_{2}, \ldots, m_{k}\right)$, so for $S$ to be co-finite we must have $\operatorname{gcd}\left(m_{1}, m_{2}, \ldots, m_{k}\right)=1$. In this case it is clear that $x^{n} \in S^{\dagger}$ if and only if $n$ can be written as a non-negative integer linear combination of $m_{1}, m_{2}, \ldots, m_{k}$. Hence, the length of the longest word not in $S^{\dagger}$ is exactly $g\left(m_{1}, m_{2}, \ldots, m_{k}\right)$.

Lastly we define the act of matching a word. Given two words $w=w_{1} \cdots w_{n}$ and $u=$ $u_{1} \cdots u_{k}$, a match of $u$ in $w$ is a subset of positions $i_{1}<i_{2}<\ldots<i_{k}$ such that $w_{i_{j}}=u_{j}$ for $j=1,2, \ldots, k$. For a given set of words $S$, a word $y$ is in $S^{\dagger}$ if and only if all positions of $y$ can be covered by a pairwise disjoint set of matches using words in $S$. In this case, we say $y$ can be matched using words in $S$. For example, if $S=\{011,012\}$, then $010121 \in S^{\dagger}$ since it can be matched using a single 011 and a single $012(\underline{01} \overline{012} \underline{1}$ or $\overline{01} \underline{01} \overline{2} 1)$. However, $01012112 \notin S^{\dagger}$ because one of the 0's will have to be matched twice to ensure the last 1 and 2 get matched.

\section{A characterization of $S$ such that $S^{\dagger}$ is co-finite}

In this section we give a complete characterization of the finite sets $S$ for which $S^{\dagger}$ is cofinite and we give some bounds on the length of a longest word not in $S^{\dagger}$. Furthermore, given a set $S$ such that $S^{\dagger}$ is co-finite, we give a detailed description on how to match a sufficiently long arbitrary word using the words in $S$ and we give lower bounds for $|S|$.

For the rest of this section, let the following be true. Let $q \geq 1$ and let $S$ be a finite set of words over an alphabet $\left\{x_{1}, x_{2}, \ldots, x_{q}\right\}$. For each $i \in\{1,2, \ldots, q\}$, let $\mathscr{T}_{i}$ denote the collection of all subsets $T_{i} \subseteq S$, where either

$$
T_{i}=\left\{x_{i}\right\}
$$

or

$$
T_{i}=\left\{x_{i}^{m_{i, 1}}, x_{i}^{m_{i, 2}}, \ldots, x_{i}^{m_{i, h_{i}}}\right\} \cup \bigcup_{\substack{j=1 \\ j \neq i}}^{q}\left\{x_{i} x_{j}^{a_{i, j}}, x_{j}^{b_{j, i}} x_{i}\right\},
$$

where $m_{i, h_{i}}>\cdots>m_{i, 2}>m_{i, 1} \geq 2, h_{i} \geq 2, \operatorname{gcd}\left(m_{i, 1}, m_{i, 2}, \ldots, m_{i, h_{i}}\right)=1$ and $a_{i, j}, b_{j, i} \geq 1$ for all $j \in\{1,2, \ldots, q\}$ such that $i \neq j$. 
Our first main result is the following.

Theorem 1. The set $S^{\dagger}$ is co-finite if and only if for each $i \in\{1,2, \ldots, q\}$, the collection $\mathscr{T}_{i}$ is non-empty.

Proof. Follows directly from Lemma 2 and Theorem 3 below.

Lemma 2. The set $S^{\dagger}$ is co-finite only if for each $i \in\{1,2, \ldots, q\}$, the collection $\mathscr{T}_{i}$ is non-empty.

Proof. Let $x_{i}$ be an arbitrary letter in our alphabet and assume $\left\{x_{i}\right\} \nsubseteq S$. If $S^{\dagger}$ is co-finite, then every sufficiently long string of $x_{i}$ 's must be in $S^{\dagger}$. So $S$ must have a string of $x_{i}$ 's, notably $x_{i}^{m_{i, 1}}$. However, then $S^{\dagger}$ will only contain strings of $x_{i}$ 's that are multiples of $m_{i, 1}$ in length. Thus $S$ must have at least two strings of $x_{i}$ 's, notably $x_{i}^{m_{i, 1}}, x_{i}^{m_{i, 2}}, \ldots, x_{i}^{m_{i, h}}$ (where $m_{i, h_{i}}>\ldots>m_{i, 2}>m_{i, 1} \geq 2$ and $h_{i} \geq 2$ ). Furthermore, every string of $x_{i}$ 's in $S^{\dagger}$ is a multiple of $\operatorname{gcd}\left(m_{i, 1}, m_{i, 2}, \ldots, m_{i, h_{i}}\right)$ in length, so if $S^{\dagger}$ is co-finite, then $\operatorname{gcd}\left(m_{i, 1}, m_{i, 2}, \ldots, m_{i, h_{i}}\right)=1$. Let $x_{j}$ be another arbitrary letter in our alphabet. Then $x_{i} x_{j}^{s}$ and $x_{j}^{s} x_{i}$ are in $S^{\dagger}$ for all sufficiently large $s$. Since $\left\{x_{i}\right\} \nsubseteq S$,

$$
\bigcup_{\substack{j=1 \\ j \neq i}}^{q}\left\{x_{i} x_{j}^{a_{i, j}}, x_{j}^{b_{j, i}} x_{i}\right\}
$$

must be a subset of $S$ where $a_{i, j}, b_{j, i} \geq 1$ for all $j \in\{1,2, \ldots, q\}$ such that $i \neq j$. Thus $\mathscr{T}_{i}$ is non-empty and the result follows.

Theorem 3. Assume for each $i \in\{1,2, \ldots, q\}$, the collection $\mathscr{T}_{i}$ is non-empty. If $\left\{x_{i}\right\} \in \mathscr{T}_{i}$, define $g_{i}=-1$ and $m_{i, 1}=a_{i, j}=b_{j, i}=0$ for all $j \in\{1,2, \ldots, q\}$. If $\left\{x_{i}\right\} \notin \mathscr{T}_{i}$, then let $T_{i}$ be a subset of $S$ of the form (3). Define $g_{i}=g\left(m_{i, 1}, m_{i, 2}, \ldots, m_{i, h_{i}}\right)$ and let $m_{i, 1}, m_{i, 2}, \ldots, m_{i, h_{i}}, a_{i, j}, b_{j, i}$ be as they are defined for the chosen $T_{i}$ for all $j \in\{1,2, \ldots, q\}$. Then $S^{\dagger}$ contains every word of length at least

$$
\sum_{i=1}^{q} g_{i}+q+(q-1)\left(\max _{i}\left(m_{i, 1}\right)\right)\left(\max _{i, j, j \neq i}\left(a_{i, j}, b_{j, i}\right)\right) .
$$

Proof. Let $y$ be a $q$-ary word of length at least

$$
\sum_{i=1}^{q} g_{i}+q+(q-1)\left(\max _{i}\left(m_{i, 1}\right)\right)\left(\max _{i, j, j \neq i}\left(a_{i, j}, b_{j, i}\right)\right)
$$

. If $|y|_{x_{i}} \geq g_{i}+1$ for all $i$, every $x_{i}$ in $y$ can be matched by shuffling the set $\left\{x_{i}\right\}$ or if $\left\{x_{i}\right\} \nsubseteq S$, by shuffling the set $\left\{x_{i}^{m_{i, 1}}, x_{i}^{m_{i, 2}}, \ldots, x_{i}^{m_{i, h_{i}}}\right\}$. Furthermore, if $|y|_{x_{i}} \leq g_{i}$ for all $i$, then $|y| \leq \sum_{i=1}^{q} g_{i}$, a contradiction. So assume WLOG that $|y|_{x_{1}} \leq g_{1},|y|_{x_{2}} \leq g_{2}, \ldots,|y|_{x_{s}} \leq g_{s},|y|_{x_{s+1}}>g_{s+1}$, $|y|_{x_{s+2}}>g_{s+2}, \ldots,|y|_{x_{q}}>g_{q}$ for some $s$ such that $1 \leq s \leq q-1$. So

$$
\sum_{i=1}^{s}|y|_{x_{i}} \leq \sum_{i=1}^{s} g_{i}
$$


which implies

$$
\begin{aligned}
\sum_{i=s+1}^{q}|y|_{x_{i}} & \geq \sum_{i=s+1}^{q} g_{i}+q+(q-1)\left(\max _{i}\left(m_{i, 1}\right)\right)\left(\max _{i, j, j \neq i}\left(a_{i, j}, b_{j, i}\right)\right) \\
& \geq \sum_{i=s+1}^{q} g_{i}+q-s+(q-1)\left(\max _{i}\left(m_{i, 1}\right)\right)\left(\max _{i, j, j \neq i}\left(a_{i, j}, b_{j, i}\right)\right) .
\end{aligned}
$$

For simplicity, let $\lambda=\left(\max _{i}\left(m_{i, 1}\right)\right)\left(\max _{i, j, j \neq i}\left(a_{i, j}, b_{j, i}\right)\right)$ and assume for all $i$ such that $s+1 \leq$ $i \leq q$, we have

$$
|y|_{x_{i}}=g_{i}+1+\gamma_{i} \lambda+r_{i},
$$

where $\gamma_{i}$ is a non-negative integer and $0 \leq r_{i}<\lambda$. It follows that

$$
\begin{aligned}
\sum_{i=s+1}^{q}|y|_{x_{i}} & =\sum_{i=s+1}^{q} g_{i}+q-s+\sum_{i=s+1}^{q} \gamma_{i} \lambda+\sum_{i=s+1}^{q} r_{i} \\
& \geq \sum_{i=s+1}^{q} g_{i}+q-s+(q-1) \lambda
\end{aligned}
$$

which implies

$$
\sum_{i=s+1}^{q} \gamma_{i} \lambda+\sum_{i=s+1}^{q} r_{i} \geq(q-1) \lambda=(s-1) \lambda+(q-s) \lambda
$$

Since $r_{i}<\lambda$ for all $i$ between $s+1$ and $q$, we have

$$
\sum_{i=s+1}^{q} r_{i}<(q-s) \lambda .
$$

So we get that

$$
\begin{aligned}
\sum_{i=s+1}^{q} \gamma_{i} \lambda & \geq(s-1) \lambda+(q-s) \lambda-\sum_{i=s+1}^{q} r_{i} \\
& >(s-1) \lambda+(q-s) \lambda-(q-s) \lambda \\
& =(s-1) \lambda
\end{aligned}
$$

Since $\sum_{i=s+1}^{q} \gamma_{i}$ is a non-negative integer, the previous inequality $\sum_{i=s+1}^{q} \gamma_{i} \lambda>(s-1) \lambda$ implies that

$$
\sum_{i=s+1}^{q} \gamma_{i} \geq s .
$$

(When reading the remainder of this proof the reader may wish to refer to Example 1 below.)

For simplicity, let $C=\left\{x_{1}, x_{2}, \ldots, x_{s}\right\}$ and $D=\left\{x_{s+1}, x_{s+2}, \ldots, x_{q}\right\}$. Note that if $x_{i} \in C$, then there exists a $T_{i}$ of the form (3) such that $T_{i} \subseteq S$. (If $x_{i} \in S$, then $g_{i}=-1$ and so $|y|_{x_{i}} \geq 0$ implies $x_{i} \in D$.) If $s<\gamma_{s+1}$, then associate all $s$ letters in $C$ with $x_{s+1}$; otherwise, associate the first $\gamma_{s+1}$ letters from $C$ with $x_{s+1}$. If $s<\gamma_{s+1}+\gamma_{s+2}$, then associate the remaining $s-\gamma_{s+1}$ letters from $C$ with $x_{s+2}$; otherwise, associate the next $\gamma_{s+2}$ letters from $C$ with $x_{s+2}$. If $s<\gamma_{s+1}+\gamma_{s+2}+\gamma_{s+3}$, then associate the remaining $s-\gamma_{s+1}-\gamma_{s+2}$ letters from $C$ with $x_{s+3}$; 
otherwise, associate the next $\gamma_{s+3}$ letters from $C$ with $x_{s+3}$. Repeat this process until every letter in $C$ has an associated letter in $D$ (which we know is possible by (4)). Once this process is completed, every letter in $C$ will be associated with exactly one letter in $D$ and every letter $x_{i}$ in $D$ will be associated with at most $\gamma_{i}$ letters of $C$. Let $x_{c}$ be a letter from $C$ and let $x_{d}$ be its associated letter in $D$ (note that $\gamma_{d} \geq 1$ by definition). By the division algorithm, we get $|y|_{x_{c}}=q_{c} m_{c, 1}+r_{c}$ where $0 \leq r_{c}<m_{c, 1}$. Consider the first occurrence of a $x_{c}$ in $y$. If it is preceded by $b_{d, c} x_{d}$ 's, then use a $x_{d}^{b_{d, c}} x_{c}$ to match the first $x_{c}$ and the first $b_{d, c} x_{d}$ 's. If not, then it must be followed by $a_{c, d} x_{d}$ 's. If that is the case, use a $x_{c} x_{d}^{a_{c, d}}$ to match the first $x_{c}$ and the first $a_{c, d} x_{d}$ 's that follow the first $x_{c}$. Now consider the second occurrence of a $x_{c}$. If it is preceded by $b_{d, c}$ unmatched $x_{d}$ 's, then use a $x_{d}^{b_{d, c}} x_{c}$ to match the second $x_{c}$ and the first previously unmatched $b_{d, c} x_{d}$ 's. If not, then it must be followed by $a_{c, d}$ unmatched $x_{d}$ 's. If this is the case, then use a $x_{c} x_{d}^{a_{c, d}}$ to match the second $x_{c}$ and the first $a_{c, d}$ unmatched $x_{d}$ 's that follow the second $x_{c}$. Repeat this process for the first $r_{c} x_{c}$ 's. This process will work because once we get to the $r_{c}$ th $x_{c}$, we still have at least

$$
\begin{aligned}
& \left(\max _{i}\left(m_{i, 1}\right)\right)\left(\max _{i, j, j \neq i}\left(a_{i, j}, b_{j, i}\right)\right)-\left(r_{c}-1\right)\left(\max \left(a_{c, d}, b_{d, c}\right)\right) \\
\geq & \left(\max _{i}\left(m_{i, 1}\right)\right)\left(\max _{i, j, j \neq i}\left(a_{i, j}, b_{j, i}\right)\right)-\left(m_{c, 1}-1-1\right)\left(\max \left(a_{c, d}, b_{d, c}\right)\right) \\
\geq & \left(\max _{i}\left(m_{i, 1}\right)\right)\left(\max _{i, j, j \neq i}\left(a_{i, j}, b_{j, i}\right)\right)-\left(\max _{i}\left(m_{i, 1}\right)-2\right)\left(\max _{i, j, j \neq i}\left(a_{i, j}, b_{j, i}\right)\right) \\
= & 2\left(\max _{i, j, j \neq i}\left(a_{i, j}, b_{j, i}\right)\right) \\
\geq & 2\left(\max _{c, d}\left(a_{c, b}, b_{d, c}\right)\right)
\end{aligned}
$$

unmatched $x_{d}$ 's. This ensures that the $r_{c}$ th $x_{c}$ is either preceeded by $b_{d, c} x_{d}$ 's or followed by $a_{c, d} x_{d}$ 's. Once we match the first $r_{c} x_{c}$ 's, use $q_{c} x_{c}^{m_{c, 1}}$ 's to match the remaining $x_{c}$ 's. This procedure will match every $x_{c}$ in $y$ and less than $\lambda x_{d}$ 's. Repeat the procedure above for every letter in $C$ with respect to their associated letter in $D$ so that every occurrence of a letter in $C$ in $y$ has been matched. Let $x_{i}$ be an arbitrary letter in $D$. After every letter in $C$ has been matched in $y$, less than $\gamma_{i} \lambda x_{i}$ 's have been matched. Thus, for each $i$ such that $s+1 \leq i \leq q$, there are at least $g_{i}+1$ unmatched $x_{i}$ 's in $y$. In which case use $x_{i}$ 's or if $x_{i} \notin S$, use the elements in the set

$$
\left\{x_{i}^{m_{i, 1}}, x_{i}^{m_{i, 2}}, \ldots, x_{i}^{m_{i, h_{i}}}\right\}
$$

to match the remaining $x_{i}$ 's in $y$. Every digit in $y$ has now been matched and therefore, $y \in S^{\dagger}$.

Remark 1. If $S^{\dagger}$ is co-finite, then for all $i$, there exists a $T_{i}$ in $S$ of the form (2) or (3). It should be noted that for any $i$, several $T_{i}$ 's of the form (3) could be in $S$ and if $\left\{x_{i}\right\} \nsubseteq S$, our choice of $T_{i}$ could impact the bound we obtain in Theorem 3 , Let $i$ be arbitrary and assume $\left\{x_{i}\right\} \nsubseteq S$. To obtain the smallest possible bound in Theorem 3 , choose a $T_{i}$ of the form (3) that includes every string of $x_{i}$ 's in $S$ (ensuring $g_{i}$ and $m_{i, 1}$ are as small as possible) and strings of the form $x_{i} x_{j}^{r}$ and $x_{j}^{s} x_{i}$ for each $j$ where $r$ and $s$ are as small as possible (ensuring $a_{i, j}$ and $b_{j, i}$ are as small as possible for all $j$ ).

The following example illustrates the procedure described in the proof of Theorem 3 , 
Example 1. Consider the set

$$
\begin{gathered}
S=\left\{x_{1}^{2}, x_{1}^{5}, x_{2}^{3}, x_{2}^{4}, x_{3}^{2}, x_{3}^{3}, x_{4}, x_{1} x_{2}, x_{2}^{2} x_{1}, x_{2} x_{1}^{3}, x_{1} x_{3}, x_{3} x_{1},\right. \\
\left.x_{1} x_{4}^{2}, x_{4}^{3} x_{1}, x_{2} x_{3}^{2}, x_{3}^{2} x_{2}, x_{3} x_{2}^{3}, x_{2}^{2} x_{3}, x_{2} x_{4}^{3}, x_{4}^{3} x_{2}, x_{3} x_{4}^{3}, x_{4}^{2} x_{3}\right\} .
\end{gathered}
$$

It can be observed that the set $S$ is of the required construction to make $S^{\dagger}$ co-finite by Theorem 3. Furthermore, we get the following values:

$$
\begin{aligned}
g_{1} & =(2)(5)-2-5=3, \\
g_{2} & =(3)(4)-3-4=5, \\
g_{3} & =(2)(3)-2-3=1, \\
g_{4} & =-1, \\
\max _{i}\left(m_{i, 1}\right) & =\max (2,3,2,0)=3, \\
\max _{i, j, j \neq i}\left(a_{i, j}, b_{i, j}\right) & =\max (1,2,3,1,1,1,1,1,2,3,0,0,2,2,3,2,3,3,0,0,3,2,0,0)=3 .
\end{aligned}
$$

By Theorem $3, S^{\dagger}$ contains every 4 -ary word of length at least

$$
3+5+1+(-1)+4+(4-1)(3)(3)=39 \text {. }
$$

Consider the word

$$
y=x_{3} x_{3} x_{4} x_{2} x_{4} x_{3} x_{3} x_{4} x_{1} x_{4} x_{2} x_{3} x_{4} x_{3} x_{4} x_{2} x_{4} x_{1} x_{3} x_{4} x_{4} x_{1} x_{4} x_{4} x_{2} x_{3} x_{4} x_{4} x_{4} x_{3} x_{3} x_{4} x_{3} x_{3} x_{4} x_{4} x_{3} x_{4} x_{2} .
$$

Since $|y|=39$, we will be able to match all the letters in $y$ using the procedure detailed in Theorem 3 above. For simplicity, let $i$ denote $x_{i}$ for all $i$ such that $1 \leq i \leq 4$. So we get

$$
y=334243341423434241344144234443343344342 .
$$

Step 1: Determine which letters go in the sets $C$ and $D$.

Since $|y|_{1}=3 \leq g_{1}$ and $|y|_{2}=5 \leq g_{2}, 1,2 \in C$. Since $|y|_{3}=13>g_{3}$ and $|y|_{4}=18>g_{4}$, $3,4 \in D$.

Step 2: Determine the associated $\gamma$ for each element of $D$.

Note that $\lambda=9$. Then

$$
|y|_{3}=13=1+1+(1)(9)+2=g_{3}+1+\gamma_{3} \lambda+r_{3} \Longrightarrow \gamma_{3}=1
$$

and

$$
|y|_{4}=18=-1+1+(2)(9)+0=g_{4}+1+\gamma_{4} \lambda+r_{4} \Longrightarrow \gamma_{4}=2 .
$$

Step 3: Associate every letter in $C$ with a single letter in $D$.

Since $\gamma_{3}=1<2=|C|$, associate only the first letter in $C$ with 3 in $D$. Thus, 1 is associated with 3 . Since $|C| \leq \gamma_{3}+\gamma_{4}$, associate the remaining letter(s) in $C$ with 4 . Thus, 2 is associated with 4.

Step 4: Match every occurrence of a letter in $C$ in $y$.

We will match the 1 's first. Note that $|y|_{1}=(1)(2)+1=q_{1} m_{1,1}+r_{1}$. In $y$, the first occurrence of a 1 is preceded by $b_{3,1}=1$ occurrences of 3 . Thus, use a 31 to match the first 3 
and the first 1 . Note that in all the lines below, a letter that is underlined is currently being matched and a letter that is overlined has been previously matched.

$$
31 \Longrightarrow \underline{3} 3424334 \underline{1423434241344144234443343344342}
$$

We have matched $r_{1} 1$ 's, so now we need $q_{1}=1$ copies of $1^{m_{1,1}}=1^{2}$ to match the remaining 1 's.

$$
11 \Longrightarrow \overline{3} 3424334 \overline{1} 42343424 \underline{13} 44 \underline{14} 4234443343344342
$$

Now we match the 2's. Note that $|y|_{2}=(1)(3)+2=q_{2} m_{2,1}+r_{2}$. The first occurrence of a 2 in $y$ is not preceded by $b_{4,2}=3$ occurrences of 4 . Thus, we use a $24^{a_{2,4}}=24^{3}$ to match the first 2 and the first three 4's that follow the first 2.

$$
2444 \Longrightarrow \overline{3} 34 \underline{24} 33 \underline{4} \underline{1} \underline{4} 2343424 \overline{1} 344 \overline{1} 44234443343344342
$$

The second occurrence of a 2 in $y$ is not preceded by three unmatched 4's. Thus, we will use another $24^{3}$ to match the second 2 and the first three unmatched 4 's that follow the second 2 .

$$
2444 \Longrightarrow \overline{3} 34 \overline{24} 33 \overline{414} \underline{2} 3 \underline{4} 3 \underline{4} 2 \underline{4} \overline{1} 344 \overline{1} 44234443343344342
$$

We have matched $r_{2} 2$ 's, so now we need $q_{2}=1$ copies of $2^{m_{2,1}}=2^{3}$ to match the remaining 2's.

$$
222 \Longrightarrow \overline{3} 34 \overline{24} 33 \overline{4142} 3 \overline{4} 3 \overline{4} \underline{2} \overline{41} 344 \overline{1} 44 \underline{2} 3444334334434 \underline{2}
$$

Step 5: Match every remaining occurrence of a letter in $D$ in $y$.

First we match the remaining 3's. Since there are at least $g_{3}+1=2$ unmatched 3's remaining, we can use the $3^{m_{3,1}}=3^{2}$ and $3^{m_{3,2}}=3^{3}$ strings to match the remaining 3 's. Since there are twelve unmatched 3's remaining, we can use six $3^{2}$ 's.

$$
\begin{aligned}
& 33 \Longrightarrow \overline{3} 3 \underline{3} \overline{24} \underline{3} 3 \overline{4142} 3 \overline{4} 3 \overline{4241} 344 \overline{1} 44 \overline{2} 3444334334434 \overline{2} \\
& 33 \Longrightarrow \overline{33} 4 \overline{243} \underline{3} \overline{4142} 3 \overline{4} 3 \overline{4241} 344 \overline{1} 44 \overline{2} 3444334334434 \overline{2} \\
& 33 \Longrightarrow \overline{33} 4 \overline{2433414234 \underline{3}} \overline{4241} \text { 는 } 44 \overline{1} 44 \overline{2} 3444334334434 \overline{2}
\end{aligned}
$$

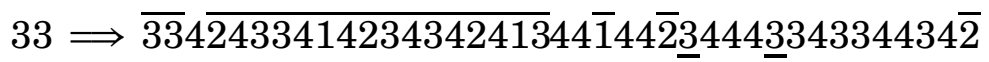

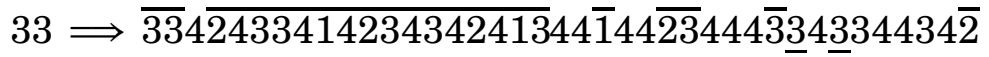

$$
\begin{aligned}
& 33 \Longrightarrow \overline{33} 4 \overline{2433414234342413} 44 \overline{1} 44 \overline{23} 444 \overline{33} 4 \overline{3} 3 \underline{3} 44 \underline{3} 4 \overline{2}
\end{aligned}
$$

Next we match the remaining 4's. Since $4 \in S$, clearly we can match the twelve remaining unmatched 4's using twelve 4's (for simplicity, the line below symbolizes repeating the process twelve times).

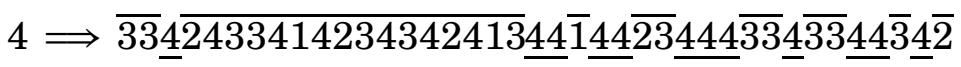

Now we have matched every letter in $y$ and it follows that $y \in S^{\dagger}$.

Corollary 4. Assume $S^{\dagger}$ is co-finite and let $n$ be the length of a longest word in the set $S$. Then the length of a longest word not in $S^{\dagger}$ is less or equal to $(2 q-1) n^{2}-(5 q-2) n+3 q-2$. 
Proof. For simplicity, let $k$ denote the length of a longest word not in $S^{\dagger}$. By Lemma 2 , for each $i \in\{1,2, \ldots, q\}$, the collection $\mathscr{T}_{i}$ is non-empty. By Theorem 3 (if $\left\{x_{i}\right\} \nsubseteq \mathscr{T}_{i}$ for some $i$, then let $T_{i}$ be a subset of $S$ of the form (3)), $k$ is at most

$$
\sum_{i=1}^{q} g_{i}+q+(q-1)\left(\max _{i}\left(m_{i, 1}\right)\right)\left(\max _{i, j, j \neq i}\left(a_{i, j}, b_{j, i}\right)\right)-1 .
$$

It is clear that $\max _{i}\left(m_{i, 1}\right) \leq n-1$ and $\max _{i, j, j \neq i}\left(a_{i, j}, b_{j, i}\right) \leq n-1$. Each $g_{i}$ is either -1 or

$$
g\left(m_{i, 1}, m_{i, 2}, \ldots, m_{i, h_{i}}\right) \leq\left(m_{i, 1}\right)\left(m_{i, h_{i}}\right)-m_{i, 1}-m_{i, h_{i}} \leq(n-1) n-(n-1)-n=n^{2}-3 n+1
$$

by (1). Since $n^{2}-3 n+1 \geq-1$ for all $n \in \mathbb{Z}$, it follows that

$$
\begin{aligned}
k & \leq \sum_{i=1}^{q} g_{i}+q+(q-1)\left(\max _{i}\left(m_{i, 1}\right)\right)\left(\max _{i, j, j \neq i}\left(a_{i, j}, b_{j, i}\right)\right)-1 \\
& \leq q\left(n^{2}-3 n+1\right)+q+(q-1)(n-1)^{2}-1 \\
& =q n^{2}-3 q n+q+q+q n^{2}-n^{2}-2 q n+2 n+q-2 \\
& =(2 q-1) n^{2}-(5 q-2) n+3 q-2 .
\end{aligned}
$$

Remark 2. The last corollary implies that the length of a longest word not in $S^{\dagger}$ is at most quadratic in the length of a longest word in $S$.

Theorem 5. Assume $S^{\dagger}$ is co-finite and let $m$ be the length of a shortest word in $S$.

- If $m=1$ then $|S| \geq q$.

- If $m=2$ then $|S| \geq q^{2}+q$.

- If $m \geq 3$ then $|S| \geq 2 q^{2}$.

In each case the bound on $|S|$ is tight.

Proof. It is clear that $S$ must contain at least one word with just $x_{i}$ 's for every $i$. It follows that $|S| \geq q$. The set $S=\left\{x_{1}, x_{2}, \ldots, x_{q}\right\}$ is a set of size $q$ such that $S^{\dagger}$ is co-finite. Assume $m \geq 2$. Then $\left\{x_{i}\right\} \nsubseteq S$ for all $i$. To ensure every sufficiently long string of $x_{i}$ 's is in $S^{\dagger}$ for all $i, S$ must have at least two strings of $x_{i}$ 's for all $i$. Furthermore, to ensure $x_{i} x_{j}^{s} \in S^{\dagger}$ for all $i, j$ and sufficiently large $s$ such that $i \neq j, S$ must contain a string consisting of a single $x_{i}$ followed by at least one $x_{j}$ for all $i$ and $j$ such that $i \neq j$. Therefore, $|S| \geq 2 q+q(q-1)=q^{2}+q$. It can be observed (by Theorem 3) that the set

$$
S=\bigcup_{i=1}^{q}\left\{x_{i}^{2}, x_{i}^{3}\right\} \cup \bigcup_{\substack{j=1 \\ j \neq i}}^{q} \bigcup_{i=1}^{q}\left\{x_{i} x_{j}\right\}
$$

is a set of size $q^{2}+q$ such that $S^{\dagger}$ is co-finite. Now assume $m \geq 3$. By Lemma 2 , for all $i \in\{1,2, \ldots, q\}$, there exists a $T_{i}$ in $S$ of the form (3). Furthermore, all of the $T_{i}$ 's are pairwise 
disjoint (i.e. if $i \neq j$, then $T_{i} \cap T_{j}=\varnothing$ ). Thus, $|S| \geq q\left|T_{i}\right| \geq q(2+2(q-1))=2 q^{2}$. It can be observed (by Theorem 3) that the set

$$
S=\bigcup_{i=1}^{q}\left\{x_{i}^{m}, x_{i}^{m+1}\right\} \cup \bigcup_{\substack{j=1 \\ j \neq i}}^{q} \bigcup_{i=1}^{q}\left\{x_{i} x_{j}^{m-1}, x_{j}^{m-1} x_{i}\right\}
$$

is a set of size $2 q^{2}$ such that $S^{\dagger}$ is co-finite.

Remark 3. Note that the lower bound on $|S|$ does not depend on $m$. This is significantly different from the case of concatenation: for $S^{*}$ to be co-finite, the cardinality of $S$ must be exponential in $m$.

\section{A prototypical case}

To find a general formula for the length of a longest word not in $S^{\dagger}$ for an arbitrary set $S$ such that $S^{\dagger}$ is co-finite is a difficult task. So instead we restrict our efforts to a family of what we deem to be the simplest constructions of $S$ such that $S^{\dagger}$ is co-finite. We define each $T_{i}$ in the following way:

$$
T_{i}=\left\{x_{i}^{m}, x_{i}^{m+1}\right\} \cup \bigcup_{\substack{j=1 \\ j \neq i}}^{q}\left\{x_{i} x_{j}^{m-1}, x_{j}^{m-1} x_{i}\right\} .
$$

This results in a set $S$ of the form

$$
S=\bigcup_{i=1}^{q}\left\{x_{i}^{m}, x_{i}^{m+1}\right\} \cup \bigcup_{\substack{j=1 \\ j \neq i}}^{q} \bigcup_{i=1}^{q}\left\{x_{i} x_{j}^{m-1}, x_{j}^{m-1} x_{i}\right\} .
$$

The cases when $q=1$ and $m=1$ are both trivial so we restrict our attention to when $q, m \geq 2$. In this section we prove that the length of the longest word not in $S^{\dagger}$ is $2 q-1$ when $m=2$ and $q m^{2}-2 q m+2 m-1$ when $m \geq 3$. We also find some elementary bounds on the number of words not in $S^{\dagger}$. For the rest of this section, unless explicitly stated, assume the set $S$ has the construction above.

Theorem 6. If $q \geq 1$ and $m=2$, the length of a longest word not in $S^{\dagger}$ is $2 q-1$.

Proof. Consider the word $y=x_{1}^{2} x_{2}^{2} \cdots x_{q-1}^{2} x_{q}$. It is clear that we cannot use a $x_{i}^{3}$ to match any letters in $y$ for any $i$. The only other words in $S$ are of even length. Since it is impossible to match a word of odd length with only even words, $y \notin S^{\dagger}$. We claim that every $q$-ary word of length at least $2 q$ is in $S^{\dagger}$. When $q=1$, clearly every unary word of length at least 2 is in $S^{\dagger}$ (since $g(2,3)=1$ ). Assume the claim holds for some $q \geq 1$. It suffices to show that the claim holds for $q+1$. Let $y$ be a $(q+1)$-ary word of length at least $2(q+1)=2 q+2$. If every letter has either 0 or at least 2 occurrences, then $y$ can be matched using the set

$$
\bigcup_{i=1}^{q+1}\left\{x_{i}^{2}, x_{i}^{3}\right\}
$$


If not, then there exists an $i$ such that $|y|_{x_{i}}=1$. Let $x_{j}$ be another letter in $y$. Then we can either use an $x_{i} x_{j}$ or an $x_{j} x_{i}$ to match the only $x_{i}$ and one of the $x_{j}$ 's. Thus, $y$ is a $q$-ary word of length at least $2 q$ (which we know is in $S^{\dagger}$ by our induction hypothesis) shuffled with a word from $S$. Thus $y \in S^{\dagger}$ and the result follows by induction on $q$.

Theorem 7. If $q \geq 2$ and $m \geq 3$, the length of a longest word not in $S^{\dagger}$ is $q m^{2}-2 q m+2 m-1$.

Proof. Follows directly from Lemmas 8,11 and 12 below.

Lemma 8. If $q \geq 2$ and $m \geq 3$, there exists a $q$-ary word $y$ of length $q m^{2}-2 q m+2 m-1$ such that $y \notin S^{\dagger}$.

Proof. Let $y=x_{1}^{m-2} x_{2}^{m-2} \cdots x_{q-1}^{m-2} x_{q}^{q m^{2}-3 q m+3 m+2 q-4} x_{1}$ and assume for the sake of contradiction that $y \in S^{\dagger}$. By observation, the only way we can match the $x_{1}$ 's is if we use $m-2$ $x_{1} x_{q}^{m-1}$ 's and a single $x_{q}^{m-1} x_{1}$. Furthermore, the only way we can match the $x_{2}$ 's is using $m-2 x_{2} x_{q}^{m-1}$ 's. We repeat this process until we match the $x_{q-1}$ 's with $m-2 x_{q-1} x_{q}^{m-1}$ 's. This leaves $q m^{2}-3 q m+3 m+2 q-4-(q-1)(m-2)(m-1)-(m-1)=m^{2}-m-1$ unmatched $x_{q}$ 's and since $g(m, m+1)=m^{2}-m-1$, it is impossible to match the remaining unmatched $x_{q}$ 's. It follows that $y \notin S^{\dagger}$.

Lemma 9. If $q \geq 1$ and $m \geq 3$, then every $q$-ary word $y$ of length at least $(q-1) m^{2}-2 q m+$ $3 m+1$ has at least one letter $x_{i}$ such that $|y|_{x_{i}} \geq m+1$.

Proof. It is clear that a longest $q$-ary word that does not contain $m+1$ occurrences of any letter is of length $q m$. The result follows if $(q-1) m^{2}-2 q m+3 m+1 \geq q m+1$. We verify that

$$
(q-1) m^{2}-2 q m+3 m+1-(q m+1)=(q-1) m^{2}-3(q-1) m=(m-3)(q-1) m \geq 0 .
$$

Lemma 10. If $q \geq 1$ and $m \geq 3$, then every $q$-ary word $y$ whose length is a multiple of $m$ and is at least $(q-1) m^{2}-2 q m+3 m$ satisfies $y=w \amalg z$, where $w$ is a word in $S^{\dagger}$ and $z$ has length exactly $(q-1) m^{2}-2 q m+3 m$.

Proof. If $|y|>(q-1) m^{2}-2 q m+3 m$ then by Lemma 9 there is some letter $x_{i}$ such that $|y|_{x_{i}} \geq m$. We can therefore match $x_{i}^{m}$ in $y$. Repeat this process until there are exactly $(q-1) m^{2}-2 q m+3 m$ unmatched letters. The matched letters form a word $w \in S^{\dagger}$ and the unmatched letters form the word $z$.

Lemma 11. If $q \geq 1, m \geq 3$ and every $q$-ary word of length $(q-1) m^{2}-2 q m+3 m$ is in $S^{\dagger}$, then every $q$-ary word of length at least $q m^{2}-2 q m+2 m$ is in $S^{\dagger}$. 
Proof. Assume that $y$ is a $q$-ary word of length at least $q m^{2}-2 q m+2 m$. Since $|y|-[(q-$ 1) $\left.m^{2}-2 q m+3 m\right] \geq q m^{2}-2 q m+2 m-\left[(q-1) m^{2}-2 q m+3 m\right]=m^{2}-m>m^{2}-m-1=g(m, m+1)$, we can write

$$
|y|-\left[(q-1) m^{2}-2 q m+3 m\right]=r m+s(m+1)
$$

for some non-negative integers $r$ and $s$. By Lemma 9, we can match letters in $y$ by making $r$ choices from $\left\{x_{1}^{m}, x_{2}^{m}, \ldots, x_{q}^{m}\right\}$ and $s$ choices from $\left\{x_{1}^{m+1}, x_{2}^{m+1}, \ldots, x_{q}^{m+1}\right\}$, leaving exactly $(q-$ 1) $m^{2}-2 q m+3 m$ unmatched letters. Let $z$ denote the word of length $(q-1) m^{2}-2 q m+3 m$ consisting of these unmatched letters. By hypothesis we have $z \in S^{\dagger}$. Therefore $y$ consists of a word in $S^{\dagger}$ shuffled with words in $S$ and so $y \in S^{\dagger}$, as required.

In the next lemma we will repeatedly apply the following procedure.

$\operatorname{MATCH}(y, i, j, r, \gamma)$ :

- Repeat $r$ times for the first $r$ occurrences of $x_{i}$.

- Consider the first unmatched occurrence of $x_{i}$ in $y$.

- If there are at least $m-1$ unmatched $x_{j}$ 's to the left of the chosen $x_{i}$ then use a $x_{j}^{m-1} x_{i}$ to match $m-1$ of these $x_{j}$ 's and this occurrence of $x_{i}$.

- If there are at least $m-1$ unmatched $x_{j}$ 's to the right of the chosen $x_{i}$ then use a $x_{i} x_{j}^{m-1}$ to match $m-1$ of these $x_{j}$ 's and this occurrence of $x_{i}$.

- Use $\gamma x_{i}^{m}$ 's to match $\gamma m$ unmatched $x_{i}$ 's.

Lemma 12. If $q \geq 2$ and $m \geq 3$, then every $q$-ary word of length $(q-1) m^{2}-2 q m+3 m$ is in $S^{\dagger}$.

Proof. By induction on $q$. First we prove the result for $q=2$. That is we prove that if $m \geq 3$ and

$$
S=\left\{x_{1}^{m}, x_{1}^{m+1}, x_{2}^{m}, x_{2}^{m+1}, x_{1} x_{2}^{m-1}, x_{2}^{m-1} x_{1}, x_{2} x_{1}^{m-1}, x_{1}^{m-1} x_{2}\right\},
$$

then every binary word of length $m^{2}-m$ is in $S^{\dagger}$.

Let $y$ be a binary word of length $m^{2}-m$. If $|y|_{x_{1}}$ is a multiple of $m$, then clearly $|y|_{x_{2}}$ is also a multiple of $m$ and it follows that $y \in\left\{x_{1}^{m}, x_{2}^{m}\right\}^{\dagger}$. So assume $|y|_{x_{1}}=\gamma m+r$ where $\gamma$ and $r$ are non-negative integers such that $0 \leq \gamma \leq m-2$ and $1 \leq r \leq m-1$. It follows that $|y|_{x_{2}}=(m-2-\gamma) m+(m-r)$.

Case 1: $1 \leq r \leq m-2-\gamma$.

Apply $\operatorname{MATCH}(y, 1,2, r, \gamma)$. Lastly, use $m-r-1-\gamma x_{2}^{m}$ 's to match the remaining $x_{2}$ 's. This process will match exactly $\gamma m+r x_{1}$ 's and $r(m-1)+(m-r-1-\gamma) m=m^{2}-m-r-\gamma m=(m-2-$ $\gamma) m+(m-r) x_{2}$ 's. This procedure is possible because once we make it to the $r$-th $x_{1}$, there are at least $(m-2-\gamma) m+(m-r)-(r-1)(m-1)=m^{2}-\gamma m-r m-1 \geq m^{2}-\gamma m-(m-2-\gamma) m-1=2 m-1$ unmatched $x_{2}$ 's ensuring the $r$-th $x_{1}$ is either preceded or followed by $m-1$ unmatched $x_{2}$ 's.

Case 2: $m-\gamma \leq r \leq m-1$.

Apply $\operatorname{MATCH}(y, 2,1, m-r, m-2-\gamma)$. Lastly, use $\gamma-m+r+1 x_{1}^{m}$ 's to match the remaining $x_{1}$ 's. This matches exactly $(m-2-\gamma) m+(m-r) x_{2}$ 's and $(\gamma-m+r+1) m+(m-r)(m-1)=$ $\gamma m-m^{2}+r m+m+m^{2}-r m-m+r=\gamma m+r x_{1}$ 's. This procedure is possible because once we 
make it to the $(m-r)$-th $x_{2}$, there are at least $\gamma m+r-(m-r-1)(m-1)=\gamma m+r-m^{2}+m+$ $r m-r+m-1=\gamma m-m^{2}+r m+2 m-1 \geq \gamma m-m^{2}+(m-\gamma) m+2 m-1=2 m-1$ unmatched $x_{1}$ 's ensuring the $(m-r)$-th $x_{2}$ is either preceeded or followed by $m-1$ unmatched $x_{1}$ 's.

Case 3: $r=m-1-\gamma$.

Subcase 1: $y$ ends with a $x_{1}$.

Apply $\operatorname{MATCH}(y, 1,2, m-\gamma-1, \gamma)$ but adjust the procedure so that once the first $m-\gamma-2$ $x_{1}$ 's have been matched, match the last $x_{1}$ as opposed to the $(m-\gamma-1)$-st. This procedure matches exactly $\gamma m+(m-\gamma-1)=\gamma m+r x_{1}$ 's and $(m-\gamma-1)(m-1)=m^{2}-\gamma m-2 m+\gamma+1=$ $(m-2-\gamma) m+(m-r) x_{2}$ 's. This procedure is possible because once we match the first $m-\gamma-2$ $x_{1}$ 's, there will be $m-1$ unmatched $x_{2}$ 's and they will all be followed by the last $x_{1}$.

Subcase 2: $y$ ends with a $x_{2}$.

Apply $\operatorname{MATCH}(y, 2,1, \gamma+1, m-2-\gamma)$ but adjust the procedure so that once the first $\gamma x_{2}$ 's have been matched, match the last $x_{2}$ as opposed to the $(\gamma+1)$-st. This procedure matches exactly $(\gamma+1)(m-1)=\gamma m+m-1-\gamma=\gamma m+r x_{1}$ 's and $(m-2-\gamma) m+\gamma+1=(m-2-\gamma) m+(m-r)$ $x_{2}$ 's. This procedure is possible because once we match the first $\gamma x_{2}$ 's, there will be $m-1$ unmatched $x_{1}$ 's and they will all be followed by the last $x_{2}$.

In every case, all the letters in $y$ can be matched using words in $S$. Thus $y \in S^{\dagger}$ and the result follows for $q=2$.

Assume the result holds for some $q \geq 2$. It suffices to show that the result holds for $q+1$. Let $y$ be a $(q+1)$-ary word of length $q m^{2}-2(q+1) m+3 m$. Assume for the sake of contradiction that $|y|_{x_{i}} \geq m^{2}-2 m+1$ for all $i \in\{1,2, \ldots, q+1\}$. Then we get

$$
\begin{aligned}
|y| & \geq(q+1)\left(m^{2}-2 m+1\right) \\
& =q m^{2}-2 q m+q+m^{2}-2 m+1 \\
& =q m^{2}-2(q+1) m+m^{2}+q-1 \\
& \geq q m^{2}-2(q+1) m+3 m+q-1 \\
& >q m^{2}-2(q+1) m+3 m .
\end{aligned}
$$

It follows that $|y|_{x_{i}} \leq m^{2}-2 m$ for some letter $x_{i}$ and WLOG we will say it is $x_{a}$. If $|y|_{x_{a}}=$ $m^{2}-2 m$, then we can match all of the $x_{a}$ 's with $m-2 x_{a}^{m}$ 's. Thus, $y$ consists of a $q$-ary word of length $(q-1) m^{2}-2 q m+3 m$ shuffled with $m-2 x_{a}^{m}$ 's. By our induction hypothesis, $y \in S^{\dagger}$ and we are done. So assume $|y|_{x_{a}}=\gamma m+r$ where $\gamma$ and $r$ are non-negative integers such that $0 \leq \gamma \leq m-3$ and $0 \leq r \leq m-1$. Let $l$ denote the number of letters in $y$ that are not $x_{a}$ 's. Then

$$
l=q m^{2}-2(q+1) m+3 m-\gamma m-r=q m^{2}-2 q m+m-\gamma m-r .
$$

By the pigeonhole principle, at least one of these letters (call it $x_{b}$ ) has at least

$$
\left\lceil\frac{q m^{2}-2 q m+m-\gamma m-r}{q}\right\rceil=\left\lceil m^{2}-2 m+\frac{m-\gamma m-r}{q}\right\rceil
$$

occurrences in $y$. If $\gamma=0$, then $\left[m^{2}-2 m+\frac{m-\gamma m-r}{q}\right\rceil \geq m^{2}-2 m+1=(m-1)^{2}$. If $\gamma \neq 0$, then $\left\lceil m^{2}-2 m+\frac{m-\gamma m-r}{q}\right\rceil \geq m^{2}-2 m+m-\gamma m-r=m^{2}-m-\gamma m-r$.

Case 1: $0 \leq r \leq m-2-\gamma$. 
Apply $\operatorname{MATCH}(y, a, b, r, \gamma)$. Once we get to the $r$-th $x_{a}$, there are at least $(m-1)^{2}-(r-$ $1)(m-1) \geq(m-1)^{2}-(m-3)(m-1)=2 m-2$ unmatched $x_{b}$ 's if $\gamma=0$ and at least $m^{2}-m-$ $\gamma m-r-(r-1)(m-1)=m^{2}-\gamma m-r m-1 \geq m^{2}-\gamma m-(m-2-\gamma) m-1=2 m-1$ unmatched $x_{b}$ 's if $\gamma \neq 0$. This ensures the $r$-th $x_{a}$ is either preceded or followed by $m-1$ unmatched $x_{b}$ 's. This procedure leaves at least $q m^{2}-2(q+1) m+3 m-(\gamma+r) m \geq q m^{2}-2(q+1) m+3 m-(\gamma+$ $m-2-\gamma) m=(q-1) m^{2}-2 q m+3 m$ unmatched letters. These unmatched letters form a word over a $q$-letter alphabet, which by Lemma 10 can be written as the shuffle of a word in $S^{\dagger}$ and a word $z$ of length exactly $(q-1) m^{2}-2 q m+3 m$. By the induction hypothesis, the $q$-ary word $z$ is in $S^{\dagger}$. It follows that $y$ is in $S^{\dagger}$ as well.

Case 2: $m-\gamma \leq r \leq m-1$.

Apply $\operatorname{MATCH}(y, b, a, m-r, 0)$. Note that since $m^{2}-m-\gamma m-r \geq(m-1) m-(m-3) m-r \geq$ $m-r$, there are at least $m-r x_{b}$ 's in $y$ ( $\gamma=0$ is omitted since it does not fall under this case). Then use $\gamma-m+r+1 x_{a}^{m}$ 's to match the remaining $x_{a}$ 's. Once we get to the $(m-r)$-th $x_{b}$, there will still be at least $\gamma m+r-(m-r-1)(m-1)=\gamma m+r-m^{2}+m+r m-r+m-1 \geq \gamma m+(m-\gamma) m-$ $m^{2}+2 m-1=2 m-1 x_{a}$ 's ensuring that the $(m-r)$-th $x_{b}$ is either preceded or followed by $m-1 x_{a}$ 's. This procedure leaves at least $q m^{2}-2(q+1) m+3 m-[(m-r) m+(\gamma-m+r+1) m]=$ $q m^{2}-2(q+1) m+3 m-(\gamma+1) m \geq q m^{2}-2(q+1) m+3 m-(m-3+1) m=(q-1) m^{2}-2 q m+3 m$ unmatched letters. As in Case 1, we can use Lemma 10 and the induction hypothesis to show that the $q$-ary word consisting of the unmatched letters is in $S^{\dagger}$, and consequently, so is $y$.

Case 3: $r=m-1-\gamma$.

Assume for the sake of contradiction that $|y|_{x_{a}}=\gamma m+(m-1-\gamma)$ and $|y|_{x_{i}} \geq m^{2}-2 m+1$ for all $i$ such that $i \neq a$. Then

$$
\begin{aligned}
|y| & \geq \gamma m+(m-\gamma-1)+q\left(m^{2}-2 m+1\right) \\
& =\gamma(m-1)+m-1+q m^{2}-2 q m+q \\
& \geq q m^{2}-2 q m+q+m-1 \\
& =q m^{2}-2(q+1) m+3 m+q-1 \\
& >q m^{2}-2(q+1) m+3 m .
\end{aligned}
$$

Thus, there exists another letter (call it $x_{c}$ ) such that $|y|_{x_{c}} \leq m^{2}-2 m$. If $|y|_{x_{c}}=m^{2}-2 m$, then you can match all of the $x_{c}$ 's with $m-2 x_{c}^{m}$ 's. Thus, $y$ consists of a $q$-ary word of length $(q-1) m^{2}-2 q m+3 m$ shuffled with $m-2 x_{c}^{m}$ 's and we are done. So assume $|y|_{x_{c}}=\delta m+s$ where $\delta$ and $s$ are non-negative integers such that $0 \leq \delta \leq m-3$ and $0 \leq s \leq m-1$. If $0 \leq s \leq m-2-\delta$, then apply Case 1 except with $x_{c}$ rather than $x_{a}$. If $m-\delta \leq s \leq m-1$, then apply Case 2 except with $x_{c}$ rather than $x_{a}$. If not, then $s=m-1-\delta$. Assume that the last occurrence of an $x_{c}$ is after the last occurrence of an $x_{a}$ in $y$. Apply $M A T C H(y, c, a, \gamma+1,0)$ but adjust the procedure so that once the first $\gamma x_{c}$ 's have been matched, match the last $x_{c}$ as opposed to the $(\gamma+1)$-st. Note that there are at least $\gamma+1 x_{c}$ 's since $\gamma+1 \geq m-3+1=m-2$ and there are at least $m-2 x_{c}$ 's by definition. This procedure is possible because once we match the first $\gamma x_{c}$ 's, there will be $m-1$ unmatched $x_{a}$ 's and they will all be followed by the last $x_{c}$. This procedure leaves at least $q m^{2}-2(q+1) m+3 m-(\gamma+1) m \geq q m^{2}-2(q+1) m+3 m-(m-3+1) m=$ $(q-1) m^{2}-2 q m+3 m$ unmatched letters. As in Case 1, we can use Lemma 10 and the induction hypothesis to show that the $q$-ary word consisting of the unmatched letters is in $S^{\dagger}$, and consequently, so is $y$. 
The case is similar if the last occurrence of $x_{a}$ is after the last occurrence of a $x_{c}$ in $y$ (just apply $\operatorname{MATCH}(y, a, c, \delta+1,0)$ but adjust the procedure so that once the first $\delta x_{a}$ 's have been matched, match the last $x_{a}$ as opposed to the $(\delta+1)$-st).

In all cases we have shown that $y \in S^{\dagger}$, so the proof is complete.

This sequence of lemmas completes the proof of Theorem 7. Given that we know the length of a longest word not in $S^{\dagger}$ for the particular family of sets $S$ studied in this section, a natural question would be to count exactly how many words are not in $S^{\dagger}$. Unfortunately, this seems to be rather difficult. Here is a rather weak lower bound.

Theorem 13. Let $t(S)$ denote the number of words not in $S^{\dagger}$. If $q \geq 2$ and $m \geq 3$,

$$
t(S) \geq \frac{q^{m^{2}+m+1}-q^{m^{2}+m}+q^{m+1}-q^{2 m+1}+q^{m}-q}{(q-1)\left(q^{m}-1\right)\left(q^{m+1}-1\right)} .
$$

Proof. Since each word in $S$ is of length $m$ or $m+1$, it follows that any word with a length that cannot be written as a non-negative integer linear combination of $m$ and $m+1$ is not in $S^{\dagger}$. It is clear that $\ell$ cannot be written as a non-negative integer linear combination of $m$ and $m+1$ if and only if $\ell=\gamma m+r$ for non-negative integers $\gamma$ and $r$ where $\gamma+1 \leq r \leq m-1$. Therefore,

$$
\begin{aligned}
t & \geq \sum_{i=1}^{m-1} q^{i}+\sum_{i=2}^{m-1} q^{m+i}+\cdots+\sum_{i=m-1}^{m-1} q^{(m-2) m+i} \\
& =\sum_{j=1}^{m-1} \sum_{i=j}^{m-1} q^{(j-1) m+i} \\
& =\sum_{j=1}^{m-1} \frac{q^{(j-1) m}\left(q^{m}-q^{j}\right)}{q-1} \\
& =\frac{q^{m^{2}+m+1}-q^{m^{2}+m}+q^{m+1}-q^{2 m+1}+q^{m}-q}{(q-1)\left(q^{m}-1\right)\left(q^{m+1}-1\right)} .
\end{aligned}
$$

This lower bound could certainly be improved by more complicated arguments, and similarly an upper bound could be calculated as well, but it seems difficult to get an exact, closed-form expression for $t(S)$.

\section{Conclusion}

One possibility for future work is to improve the upper bound given in Theorem 3, Another issue not addressed in this paper concerns the computational complexity of the following problem: Given a set of words $S$ such that $S^{\dagger}$ is co-finite, what is the length of a longest word not in $S^{\dagger}$ ? We have given an upper bound for this length but we have not given an algorithm to determine it exactly. Returning for a moment to the classical Frobenius problem, we note that Ramírez-Alfonsín showed that computing the Frobenius number for 
a given set of integers is NP-hard with respect to Turing reductions [5]. We also claimed in our introduction that the classical Frobenius problem is equivalent to the special case of the problem considered in this paper where the set $S$ is over a unary alphabet. However, this is not entirely true from the point of view of computational complexity. In the classical setting, the size of the inputs $m_{1}, m_{2}, \ldots, m_{k}$ would be measured in terms of the lengths of their base-2 representations (i.e., in terms of their base-2 logarithms); in our setting, these integers are represented as the unary strings $0^{m_{1}}, 0^{m_{2}}, \ldots, 0^{m_{k}}$. With respect to this unary representation, the classical Frobenius problem is solvable in polynomial time. Over larger alphabets, however, we don't know what the computational complexity is of determining the length of a longest word not in $S^{\dagger}$.

\section{References}

[1] A. Brauer, On a problem of partitions, Amer. J. Math. 64 (1942), 299-312.

[2] S. Eilenberg, S. MacLane, On the groups of $H(\Pi, n)$. I., Annals of Mathematics (2) 58 (1953), 55-106.

[3] M. Jantzen, The power of synchronizing operations on strings, Theoret. Comput. Sci. 14 (1981), 127-154.

[4] M. Jantzen, Extending regular expressions with iterated shuffle, Theoret. Comput. Sci. 38 (1985), 223-247.

[5] J. L. Ramírez Alfonsín, Complexity of the Frobenius problem, Combinatorica 16 (1996), 143-147.

[6] J. L. Ramírez Alfonsín, The Diophantine Frobenius Problem, Oxford University Press, 2005.

[7] M. Warmuth, D. Haussler, On the complexity of iterated shuffle, J. Computer and System Sciences 28 (1984), 345-358.

[8] Z. Xu, The Frobenius Problem in a Free Monoid, Ph.D. thesis, University of Waterloo, 2009. 